\section{Rembrandt: estética, sujeción y corporalidad}

\author{
Juan Alberto Di Loreto *
}

Resumen: La producción y el contexto del pintor holandés Rembrandt Harmenszoon van Rijn, permite un análisis de diversos cambios en las concepciones de la subjetividad y el cuerpo que se dan en el mundo europeo a comienzos del siglo XVII. A través de las llamadas lecciones de anatomía y sus autorretratos, Rembrandt da cuenta de la racionalización, la aparición del individuo y objetivación de la corporalidad: de un cuerpo unido a la comunidad en la Edad Media, a la representación en la Modernidad de un cuerpo como un objeto inerte; del sujeto comunitario a la percepción del sujeto como unidad.

Palabras clave: subjetividad - Rembrandt - Harmenszoon van Rijn - 1606-1669 - arte barroco - modernidad - estética.

[Resúmenes en inglés y portugués en la página 139]

${ }^{(*)}$ Licenciado en Ciencias de la Comunicación (UBA) y Bibliotecario (Biblioteca Nacional Mariano Moreno), ejerció como docente en Comunicación y Ciencia Política (UBA). Con el foco puesto en el cruce entre el arte, la comunicación y la filosofía colaboró en periódicos y revistas de Buenos Aires y el interior. Actualmente se desempeña como Bibliotecario en la Universidad de Palermo.

Vida, obra, sucesos, ideas: Rembrandt. Un nombre que refiere al emblemático artista barroco pero también al despertar de una época. Porque este significante, "Rembrandt", nos aparece delimitado sincrónicamente por su contemporaneidad, pero (re)significado diacrónicamente por la posteridad. Su vida, sus decisiones, su contexto, su co-texto, su forma de mirar y pintar. En definitiva: qué significó la producción de este artista, qué signos nos dejó que podemos seguir significando.

Pero hay más: el conjunto significante Rembrandt, que podemos agrupar bajo su obra pictórica, reenvía al Sujeto moderno. Así, en mayúsculas, en su sentido fuerte: Sujeto, el Yo, el Cogito cartesiano, el substrato. Porque su obra, por qué tardar en decirlo, es parte de aquel entramado que llamamos Modernidad. En palabras de Eduardo Grüner (2005, p. 17): "es parte de la memoria de la especie, todo un sistema de representaciones que implica a los sujetos sociales en sus representaciones, prácticas y supuestos ideológicos". Pero seamos sinceros, más allá de todo: esto no es más que una interpretación, una búsqueda de causas y efectos entre el relato de una vida, unas prácticas, una sociedad, lecturas filo- 
sóficas e históricas. Al fin y al cabo, un sesgo en el (infinito) entramado semiótico de las interpretaciones.

\section{Rembrandt}

Para comprender mejor la nueva representación del cuerpo humano y la construcción social de la subjetividad que se estaba realizando en el comienzo de la Modernidad a través de la obra artística de Rembrandt, es conveniente hacer una introducción biográfica al personaje, su época y su espacio; en definitiva, situarlo en un contexto determinado para ver sus posibilidades y limitaciones.

Rembrandt Harmensz van Rijn ${ }^{1}$, penúltimo de nueve hijos, nació en la ciudad de Leiden el 15 de julio de 1606, y murió en Ámsterdam en el año de 1669. Leiden, ubicada en la Holanda meridional, cosechó los frutos de una nación pujante gracias al colonialismo y el poderío marítimo, lo que tuvo por efecto "un rápido desarrollo cultural" (Rosenberg, 1987, p. 16). Leiden no sólo era un centro industrial (especializado en tela), sino que poseía una de las universidades más prestigiosas y afamadas de la época.

Sus padres eran lo que se pueden considerar burgueses acomodados o, al menos, con un buen pasar económico. Por un lado su padre, Harmen Gerritszoom, poseía un molino en las puertas de la ciudad cercano al viejo río Rhin. Por otro lado su madre, Cornelia van Suydtbroeck, descendía de una familia del patriciado urbano. Estas condiciones familiares posibilitaron que Rembrandt fuera el único de los nueve hijos que accedió a una notable educación.

En efecto, a los siete años fue enviado a la Escuela Latina (Rosenberg, 1987, p. 20) donde se instruyó en el latín (a través de la lectura de los clásicos como Cicerón o Virgilio) y el griego. En las clases de religión estudió la Biblia y aprendió la doctrina calvinista.

Así, en el próspero y pujante ambiente de la burguesía holandesa crecería el joven Rembrandt. Recordemos que Holanda junto con Inglaterra fueron las potencias que menos sintieron los efectos de la recesión económica que se produjo a fines del siglo XVI, causada básicamente por la poca disponibilidad de metales preciosos, la baja de precios y la reducción de intercambios comerciales. Esta situación tuvo como efecto la decadencia de las potencias ibéricas; en cambio, Holanda e Inglaterra comenzaron su camino hacia la hegemonía comercial mundial.

Este contexto económico de progreso, que se cristalizó definitivamente en el siglo XVIII y XIX con los logros políticos de la burguesía, ayudó a configurar los nuevos elementos que Rembrandt traería al terreno artístico. Esto es, que las nuevas representaciones que se daban en el terreno de la pintura y el dibujo fueron posibles, en parte, gracias a las concepciones más liberales que traía la burguesía respecto de la limitada Edad Media.

Pero si bien en lo profesional Rembrandt se había convertido en toda una celebridad y había creado su propia escuela artística, cuyos alumnos reproducían fielmente el estilo del maestro, en lo personal su vida era por completo desdichada.

El artista contrajo matrimonio con Saskia van Uylengurgh en 1634, joven perteneciente a las prósperas clases acomodadas. Esto permitió a Rembrandt ascender en la escala social y afianzar más su fama de pintor. Pero a la felicidad del comienzo de la relación le siguió 
la tragedia, ya que de los cuatro hijos que tuvo con Saskia sólo el último, Titus, sobrevivió a la infancia. Luego vinieron dos niñas en 1638 y 1640, ambas llamadas Cornelia, que no sobrevivieron más de un mes. Dos años más tarde en 1642, moría en Ámsterdam la propia Saskia ${ }^{2}$.

En cuanto a lo estrictamente artístico, Rembrandt es, en primer lugar, uno de los maestros del claroscuro, estilo impulsado por Caravaggio en el barroco temprano (Rosenberg, 1987 p. 308) que juega con las luces y las sombras para tratar de conseguir ciertos efectos dramáticos e inquietantes en sus pinturas y dibujos.

En segundo término, lo podemos ubicar como uno de los representantes del barroco centroeuropeo en donde se destacará, entre otras cosas, un carácter mercantil de lo artístico. Pero en contraposición al barroco cortesano, más ligado a los intereses del catolicismo romano, Rembrandt desarrollará en Holanda "un barroco más carnal" (Aulicino, 2006, párr. 4) y sobre todo, realista. Aunque el estilo de algunas de sus obras no coincidían del todo con el gusto burgués. Así es como, en ocasiones, su "naturalismo extraño era rechazado" (Aulicino, 2006, párr. 9) porque los ricos burgueses no querían ser retratados en una atmósfera enrarecida.

En tercer lugar, Rembrandt fue uno de los que más trabajó sobre el retrato de grupo, generalmente encargado por cofradías de profesionales o gremios, como sería el caso de las lecciones de anatomía que se verán más adelante. En estos retratos colectivos pintaba a un gran grupo, de parecida edad y vestido, colocados en filas para que todos puedan verse retratados puesto que compartían los gastos ${ }^{3}$.

\section{Cuerpos Medievales y Modernos}

Ahora bien, una vez situado el personaje en la dimensión histórica y estética, es pertinente ver qué concepción de la corporalidad imperaba hasta la ruptura que impuso la Modernidad y que podemos ver representada por medio de artistas de la talla de Rembrandt o Caravaggio. Ruptura que se va a manifestar en el lento pero inexorable desgarramiento del tejido social que había constituido la Edad Media.

En este sentido, hubo un tiempo en que el cuerpo era la viva expresión del pueblo. Mejor dicho, el cuerpo era inseparable de la comunidad, del mundo, del cosmos. Sin límites hacia el otro y desenfrenado en sus funciones orgánicas. El cuerpo grotesco -nos dice Le Breton citando a Mijail Bajtín- es uno abierto al mundo, es decir: "en los orificios, en las protuberancias, en todas las ramificaciones y excrecencias" (Le Breton, 2002 p. 31). Este cuerpo, que Bajtín denomina "grotesco" y que en este trabajo podemos nominar como medieval o cuerpo comunitario, "no tiene una demarcación respecto del mundo, no está encerrado, terminado ni listo, sino que se excede a sí mismo, atraviesa sus propios límites" (Le Breton, 2002 , p. 31). Todavía no estaba definido, recortado, por una matriz social constrictiva.

Este cuerpo comunitario o integrado al mundo tenía su máxima expresión en el carnaval medieval, en donde el pueblo daba rienda suelta al desparpajo en oposición a la seriedad y sobriedad impuesta por la estructura eclesiástica que colonizaba todos los ámbitos de la vida. En efecto, "en el júbilo del carnaval, por ejemplo, los cuerpos se entremezclaban sin distinciones, participan de un estado común: el de la comunidad llevado a la incandes- 
cencia" (Le Breton, 2002 p. 30). En este marco festivo era impensable una separación, en el sentido de espectáculo, todos eran parte del escenario, todos participaban, nadie miraba. Pero este tipo de prácticas sociales, que se pueden reconstruir a través de obras como la de Rabelais, hay que insertarlas en un contexto social determinado.

Primero, como se dijo, hay que pensar en un mundo dominado espiritualmente por la estructura que despliega la Iglesia Cristiana en toda la Europa Occidental, convirtiéndose en la cultura oficial de la época. En consecuencia, en segundo lugar, el cuerpo humano era -junto con todos los bienes que habitaban la existencia- una creación de Dios; lo cual le aseguraba al cuerpo un destino que sólo podía ser manipulado por la Iglesia. En tercer lugar, todavía se concebía al mundo con restos del viejo animismo. En este enfoque, la vida lo inundaba todo, ya que todavía "no se había descubierta (la) mera materia, es decir, realmente inanimada, muerta" (Jonas, 2000 p. 21). En contraposición a la Modernidad (objetivante), para el hombre anterior a la Ilustración la vida era la regla del universo y "la muerte es el enigma que mira fijamente a los ojos al hombre y contradice ese fenómeno comprendido, autoexplicativo, natural, que es la vida universal" (Jonas, 2000 p. 22).

Así, se puede apreciar como un tejido social como el medieval no pudo haber producido una mirada instrumental y calculante sobre el cuerpo como la que representará Rembrandt en el siglo XVII. Fundamentalmente, porque no pudo separar al cuerpo humano del mundo circundante, porque no pudo convertirlo en res; en definitiva, re-ificarlo o cosificarlo para transformarlo en un mero instrumento. Claro que, para que surgiera una visión semejante, debía entrar en juego en la escena social una nueva clase social con nuevos intereses y prácticas sociales.

Con el establecimiento de la burguesía y del Capitalismo como modo de producción dominante, se despliega un novedoso proceso civilizatorio que tendría como efecto retraer la corporalidad en sus funciones orgánicas, que lo limitaría como una frontera, pero pondría toda su atención en la psicología y en las buenas costumbres pero también en su disciplinamiento. Así, el cuerpo era algo que había que reprimir y contener. Según Nobert Elías, cuando el hombre deja de estar solo y crecen sus relaciones sociales, su interdependencia con los demás, "se ve obligado a organizar su comportamiento de modo cada vez más diferenciado, más regular y más estable" (Elías, 1994 p. 451). En este sentido, la corporalidad se comienza a mostrar como algo que había que dominar y ciertas reacciones empezaron a ser consideradas como irracionales o incivilizadas. Las pasiones retrocedieron ante la mirada de los otros que lo comenzaron a condicionar. A su vez, una parte importante del aplacamiento de los instintos es activada por el individuo inconscientemente. Elías denomina autocoacción a este mecanismo de control, cuya función es la regulación permanente que se le inculca al individuo desde que nace.

\section{Amanecer de una nueva estética}

“...el arte es, dentro de la cultura afirmativa, el ámbito supremo y más representativo de la cultura". (H. Marcuse, Cultura y sociedad) 
Se pasó, entonces, de un cuerpo abierto al mundo a uno cerrado sobre sí mismo. Pero en realidad, ¿qué significa esto? O mejor dicho: ¿qué relevancia puede tener este hecho a los efectos de un trabajo como el que aquí se propone?

Lo que se intenta mostrar es cómo una incipiente época (la burguesa) va a construir ciertas representaciones y uno de los que va a cristalizar estos nuevos signos no es otro que el viejo Rembrandt. El artista holandés parece convertirse en un punto en la historia del arte en donde los restos de la Edad Media buscan sobrevivir entre las nuevas "cosas del mundo" que mueven a los hombres a realizar su trabajo. Como fuente de inspiración, los motivos religiosos dan paso a los deseos propios de los individuos que quieren verse retratados, así como las nuevas profesiones y prácticas sociales.

En otras palabras, la nueva matriz social construye una estética que le es propia y que va a coincidir con las necesidades y usos de un sujeto capitalista que iba a transformar al mundo de una forma nunca antes vista. Esto va a traer como efecto que la estética "sacra" y de inspiración exclusivamente bíblica va a ir quedando relegada, puesto que el centro de la escena va a ser ocupado por el ser humano. Pero este fenómeno no va a ser exclusivo de la pintura, sino que se va a dar en otros ámbitos como en el pensamiento filosófico, por ejemplo en autores renacentistas como Tomás Moro, o en pensadores políticos como Nicolás Maquiavelo.

Estas necesidades y usos tienen que ver con que, como ya se ha sugerido, Dios y la Iglesia ya no van a constituir el centro de la vida social. Es en ese punto que las nuevas formas de socialización harán que el individuo desee verse representado en las distintas formas plásticas. Ya no es la comunidad, sino el individuo recortado y toda su geografía la que comienza a representarse. En efecto, la boca, "órgano de la avidez del contacto con los otros por medio del habla, del grito o del canto", del exceso, de la bebida y de la comida (Le Breton, 2002, p. 41), deja su lugar de privilegio al aspecto más psicológico de los retratados: la mirada. En definitiva, lo que sucede es que "la axiología corporal se modifica. Los ojos son los órganos que se benefician" con la emergencia de la nueva cultura del individuo (Le Breton, 2002, p. 30). En el caso de Rembrandt esta importancia de la mirada en general, y de los ojos en particular, se torna curiosa: si se observa detenidamente distintas obras del artista todos los ojos que tienen los personajes son los mismos. Desde Saskia, su mujer, en un retrato (Retrato de Saskia con sombrero, óleo sobre tabla, 1633) hasta la figura de Abraham en una representación de una escena bíblica (El sacrificio de Isaac, óleo sobre lienzo, 1635). Pero tampoco escapan a este rasgo particular los retratos colectivos ni los propios autorretratos de Rembrandt.

En este sentido, el de Rembrandt es un caso paradigmático, pues la realización de autorretratos recorre una parte importante de su obra. Lo que nos muestra la importancia que había adquirido como valor la propia representación, como parte del ejercicio artístico, pero también como muestra de una nueva sensibilidad singular. Es así como se ha señalado que su gran producción de autorretratos ${ }^{4}$ no es sino "su conciencia de individuo único e irrepetible y su inexorable caminar hacia la muerte" (Parrondo, 2005, p. 11). Por tanto, la visión cristiana del "más allá" y de la promesa de otra vida se quiebra por completo. El artista en general no pinta y se pinta porque tiene un lugar asegurado junto al creador, sino justamente por lo opuesto: al no tener una existencia asegurada y experimentar como hombres de carne y hueso "el sentimiento trágico de la vida" (Unamuno, 1993, p. 23) y, por 
eso mismo, lo absurdo de la existencia como diría Albert Camus ${ }^{5}$, eso es lo que lo motiva a realizar la obra. "Uno pinta y escribe porque se sabe finito, mortal y no por otra cosa". Si la existencia fuese algo completo, el arte no tendría sentido. Para qué intentar trascender si tengo la eternidad por delante.

Sin embargo, en el primer período de autorretratos de Rembrandt, lo que predomina es sobre todo una búsqueda técnica centrada en la expresión y la fisonomía, en donde no importa los temas sino el claroscuro y los estados de ánimo. Por ejemplo, el primer autorretrato que se le conoce es Autorretrato con pelo enmarañado (Óleo sobre tabla, hacia 1628), en donde la atención no está puesta tanto en su persona sino más bien en un ejercicio plástico que tiene que ver con el estudio "de la incidencia de la luz sobre un rostro visto como de improviso" (Parrondo, 2005, p. 29).

En el período de Leiden, en cambio, los temas comienzan a tomar una relevancia mayor. Por ejemplo, El pintor en su estudio (Óleo sobre tabla, 1629) se lo interpreta como un cuadro en donde Rembrandt simboliza su punto de vista sobre el trabajo constante del artista. También, apelando a retratos de artistas célebres que se hacían en el siglo XVI, Rembrandt aparece en numerosos autorretratos disfrazados o con trajes históricos (vestido de soldado o con traje de burgués o como un noble oriental).

Hacia los últimos períodos de su carrera, como en Autorretrato con bastón (Óleo sobre lienzo, 1658) y Autorretrato con paleta y pinceles (Óleo sobre lienzo, 1665), Rembrandt muestra una gran conciencia de su existencia al representar el paso del tiempo de su propio cuerpo, ahora cansado, pero también con cierto aire melancólico. Esto lo vemos en particular en la "sombra que vela la mirada del artista" (Parrondo, 2005 p. 144).

Por último, podemos concluir en este apartado que Rembrandt aparece como muestra de lo que estaba sucediendo históricamente con las representaciones que el hombre se encargaba de crear. En efecto, "el individuo deja de ser el miembro inseparable de la comunidad, del gran cuerpo social, y se vuelve un cuerpo para él solo" (Le Breton, 2002, p. 43). El hombre aislado, recortado del tejido social, es lo que toma fuerza en esta época y tiene su primer centro en el rostro del hombre, que se convierte en la marca clave de su singularidad. Es por eso que a partir del siglo XV, el retrato como forma pictórica en Europa se afianza en el arte. Así, al no tener un anclaje religioso, la obra de arte se vuelve "una celebración personal sin ninguna otra justificación" (Le Breton, 2002, p. 42).

Desde este punto de vista, "el arte por el arte por el arte" se hace posible, puesto que su derecho a existir ya no giraría en torno a Dios y sus administradores terrenales. Es por eso que, como bien lo dice Adorno, "tras haber sacudido su función cultual, (...) la autonomía exigida por el arte se alimentó de la idea de humanidad" (Adorno, 1983, p. 9).

\section{Liberación y sujeción}

"La sociedad burguesa liberó a los individuos, pero sólo en tanto personas que han de mantenerse disciplinadas"

(H. Marcuse, Cultura y sociedad). 
Pero no hay que engañarse: la autonomía (del arte) aquí no es sinónimo de liberación. Así lo confirma Adorno cuando dice que, si bien se alimenta de la idea de humanidad, esta misma idea se desmorona "en la medida en que la sociedad se fue haciendo menos humana" (Adorno, 1983 p. 9). Dicho de otro modo, el arte consigue una liberación del encierro eclesiástico, pero sólo para caer en las invisibles manos del mercado de bienes. $\mathrm{Al}$ mismo tiempo, va a ir construyendo una autonomía -como le sucede al sujeto humanode la propia comunidad que lo produce; la extensa marcha del realismo hasta lo abstracto, puesto que el divorcio con las cosas de la sociedad se va a dar fuertemente en el terreno de la referencialidad. Se abandona esa obligación de "reflejar" y estar atado al mundo para dar paso a la pura expresión del artista.

Volvamos a Rembrandt y a la corporalidad.

Una vez librado del marco cultural y cultual que dictaba la Iglesia, el arte comenzó a desplegar toda una serie de nuevas representaciones. Como se observó antes, las figuras bíblicas van dejando su lugar al sujeto (individual) que arriba con la Modernidad y el capitalismo. El arte vira hacia lo humano. Aunque se puede preguntar: ¿el arte vira hacia lo humano o es que ocurre ese movimiento contradictorio que afirmaba Adorno: liberación de la función cultual y entrada en una fase de deshumanización de la sociedad? ¿Acaso no era humana la representación con motivos religiosos?

Lo que ocurre en esta operación, en este movimiento histórico del arte es, en definitiva, una sujeción: el cuerpo de los seres humanos es capturado por las representaciones del nuevo arte burgués. Captura, ¿por qué ocurre tal operación simbólica en los albores de la Modernidad? ¿Por qué se da en ese momento y no en otro? Trasladado a la dimensión "Rembrandt", que es lo que aquí importa, se puede preguntar lo siguiente: entonces, ¿qué significa exactamente la pintura La lección de anatomía del doctor Tulp en el contexto de una reflexión como ésta?

Recorramos primero La lección de anatomía del doctor Tulp (óleo sobre lienzo, 1632), que es encargada a Rembrandt por miembros del gremio de cirujanos "que querían hacerse retratar en grupo en el contexto de una clase de anatomía" (Parrondo, 2005, p. 35). La pintura nos muestra una estética realista y racionalista en una escena donde vemos a los aprendices atentos a la operación que el doctor Nicolás Tulp, profesor de anatomía del gremio de cirujanos entre 1628 y 1653, realiza sobre un cuerpo inerme y, ahora, al servicio de las luces de la Ilustración que asomaban por esos años en toda Europa.

Pero el cuerpo sometido al ojo clínico no es un cuerpo cualquiera. Adriaan Adrianszoon, conocido como Aris Kint, no era un burgués ni un artesano, sino un vulgar ladrón. La sujeción y el estudio quirúrgico del cuerpo unen sus fuerzas y, como no podía ser de otra manera, comienzan sus indagaciones por los "descartes sociales" ${ }^{\text {. }}$ Pero esto no es una casualidad, y se lo ha interpretado de la siguiente manera: "La escena de la disección es, pues, ejercicio de jurisdicción sobre el cuerpo de Aris Kindt, acto de dominación penal y soberana que al mismo tiempo resulta ejemplar y sustantivo, simbólico y material" (Barker, 1984, p. 95).

La nueva forma cultural muestra las uñas. Los cuerpos ya no tendrán derecho a la trascendencia ni a poder descansar en paz. El cuerpo se convertirá en objeto del más extraordinario dispositivo sin el cual es imposible pensar la Modernidad: el Estado-Nación. Ya sea en el ejército, en los hospitales o las cárceles el cuerpo será un blanco susceptible de ser 
controlado, marcado, estudiado, medido, curado. En definitiva, lo que se buscaba era la docilización del cuerpo ${ }^{7}$.

Pero este sometimiento no es gratuito, puesto que ahora el cuerpo estará inmerso en un "campo político": "las relaciones de poder operan sobre él una presa inmediata; lo cercan, lo marcan, lo doman, lo someten a suplicio, lo fuerzan a unos trabajos, lo obligan a unas ceremonias, exigen de él unos signos" (Foucault, 1987, p. 32). Eso mismo le sucede a Aris Kindt, las prácticas del doctor Tulp, y del mismo Rembrandt en otro aspecto, lo someten a constituir un medio para el avance de la moderna medicina, pero, a su vez, lo obligan a significar (por última vez) bajo un "régimen de sujeción que se empieza a practicar en esta pintura" (Barker, 1984, p. 94).

La ley y la ciencia, aún muerto Aris Kindt, no lo deja tranquilo. Por eso es que la obra no celebra su cuerpo, sino el acto de conocimiento que desarrolla Tulp a su alrededor y delante de sus aprendices. Así, es como el cuadro va a representar no una anécdota histórica (la disección pública de cadáveres tan practicada por aquellos años, que era seguida por una comida), sino un "episodio cargado con la semiosis del nuevo poder" (Barker, 1984, p. 94). Y, al fin y al cabo, no asistimos a otra cosa que a la representación que una clase social realiza sobre sí misma.

Cabe agregar que, a la mirada quirúrgica y burguesa sobre la corporalidad, se estaba sumando también en tierras holandesas la mirada filosófica bajo la pluma del padre del racionalismo: René Descartes ${ }^{8}$; quien inscribe en lo corporal todos los errores e infortunios del hombre, puesto que él es -como declara en sus Meditaciones Metafísicas-primero una "cosa que piensa" más que una res extensa. Con esta visión filosófica por detrás, se deja el camino libre para hacer del cuerpo, antes que nada, una máquina para el trabajo.

Nace, entonces, una corporalidad más ligada a una sujeción que a una liberación. Porque, como ya sabemos, no hay tal libertad (absoluta) sino en formas de infinitas de alienación?

\section{La otra lección de anatomía}

La otra referencia importante que encontramos respecto de la nueva corporalidad que se estaba construyendo en las representaciones pictóricas de Rembrandt, es La lección de anatomía del doctor Deyman (óleo sobre lienzo, 1656). Pero esta obra no alcanzó la fama de La lección de anatomía del doctor Tulp. Quizás, porque un incendio destruyó parte importante del lienzo en 1723, del cual se conserva el fragmento de la parte central de la zona inferior del cuadro. Allí sólo podemos observar a Gysbrecht Calcoen, maestro del gremio de cirujanos, participando de la disección de un cadáver con la calota ${ }^{10}$ en su mano izquierda. Pero del protagonista de la pintura, el doctor Jay Deyman, sólo se puede apreciar el pecho y las manos operando sobre el cerebro del muerto, ya que su cabeza fue víctima del incendio mencionado.

Se ha señalado que lo interesante y original de esta obra es la posición del cuerpo diseccionado: se encuentra de frente a la mirada del espectador. También se lo ha visto como una cita al Cristo muerto de Andrea Magenta (Parrondo, 2005). Pero para los ojos de los habitantes del siglo XXI, esa imagen del cuerpo muerto que interpela de frente al observador no puede ser otra que la del cadáver de Ernesto "Che" Guevara. Claro que, la imagen de 
Guevara muerto en Bolivia, está rodeada para nuestra mirada cultural con los signos del arrojo y la revolución social de épocas pasadas. En el caso de la pintura de Rembrandt, lo que rodea al cuerpo es la mirada científica y de progreso de los inicios de la Modernidad.

\section{La imposibilidad}

Lejos del siglo XVII que nos trae Rembrandt con su excepcional obra, la corporalidad y la estética en la actualidad constituyen temas centrales de nuestras sociedades occidentales. Con la pérdida del Estado-Nación como articulador de nuestras vidas y, junto con esto, la destitución de los conceptos de Verdad, Progreso y Razón, los temas en el campo teórico viran hacia temas como el cuerpo y la sexualidad, la administración de la bios (la biopolítica). Cuando la Identidad no puede anclarse en ideas e ideales trascendentes (que ya no hacen ningún sentido), cuando el peligro de la disolución y de la dispersión total de las subjetividades se convierte en un signo epocal, cuando todo esto se condensa, el Cuerpo y su cuidado y su estética se convierten en el centro de nuestras vidas. En palabras de Terry Eagleton: "El sujeto posmoderno, a diferencia de su antecesor cartesiano, es uno cuyo cuerpo está integrado a su identidad" (Eagleton, 1998 p. 109). Somos el cuerpo que representamos en nuestros avatares sociales. Es decir, al derrumbarse todo a su alrededor, el sujeto contemporáneo queda desamparado y sin referencias fijas y fuertes a las que remitirse. A lo único que puede recurrir es a la inmediatez de su cuerpo, con la ilusión de poder controlar algo en un mundo tan fragmentado.

Pero la cuestión es que las representaciones estéticas del cuerpo construidas en la actualidad tienen su centro en un discurso publicitario y cuya gramática se fluidiza y transforma en las redes sociales. La liberación y la exhibición de los cuerpos no es total, ya que "si el cuerpo libidinal sirve, el trabajador, no" (Eagleton, 1998, p. 112). El eje de las representaciones parece desplazarse políticamente de la producción al placer: "Hay cuerpos mutilados en abundancia, pero pocos desnutridos" (Eagleton, 1998, p. 112). Hay una corporalidad liviana que acompaña la estética de esta era de vacío.

Ahora bien, la obra de Rembrandt nos sirve para comprender no tanto la larga marcha que ha sufrido el cuerpo como representación desde la lejana Edad Media hasta el ocaso de la Postmodernidad, sino cómo fueron los comienzos de una concepción de la corporalidad. También el artista holandés sirve de muestra para observar una etapa clave de la historia de la subjetividad: el momento en que el hombre empieza a tomarse a él mismo como tema y referencia ${ }^{11}$.

El arte de Rembrandt, entonces, parece operar como signo del cambio que estaba sucediendo con la existencia del hombre. Pero también -como veíamos en los sucesivos autorretratos del pintor- como conciencia del propio devenir del ser humano, en esa "carrera que nos precipita un poco más hacia la muerte" y en la que "el cuerpo mantiene una delantera irreparable" (Camus, 2004, p. 16). Conciencia (exasperada) que prevalece en una época como la nuestra tan empeñada (por medio de la tecnología, de la techné) en preservar del paso del tiempo lo imposible: el cuerpo propio. 


\section{Notas}

1. Literalmente: "junto al Rhin".

2. Cabe mencionar también, luego de la muerte de Saskia, las penurias económicas sufridas por el artista (entra en bancarrota) y los "escándalos morales" que lo envolvieron por el desarrollo de su vida privada.

3. En diversas ocasiones, luego de ver la obra terminada, los que pagaban por las pinturas le reclamaban al artista por ciertos detalles o por el hecho de no aparecer protagonizando la composición.

4. Exactamente se pueden contabilizar el siguiente número de autorretratos: 30 pinturas, 26 aguafuertes y 12 dibujos.

5. Dice Camus: "por una parte, lo absurdo enseña que todas las experiencias son indiferentes y, por la otra, impulsa a la mayor cantidad de experiencias" (Camus, 2004, p. 69). Una de esas experiencias, la constituye el Arte, como expresión contradictoria del ser de lo humano.

6. Recordemos que recién en esta época se comienzan a hacer disecciones de cadáveres permitidas. Años atrás estas prácticas sólo se realizaban en forma clandestina

7. Aquí, con Michel Foucault, entendemos por dócil a "un cuerpo que puede ser sometido, que puede ser utilizado, que puede ser transformado y perfeccionado" (Foucault, 1987, p 140).

8. Recordemos que cuatro años antes (1628) de que Rembrandt pintara la lección de anatomía Descartes se trasladaba a Holanda en busca de "soledad y libertad para crear" (Descartes, 2004).

9. Como alienación no entendemos algo puramente negativo, sino en el sentido ontológico como un estado del hombre. En términos fenomenológicos: no hay un sujeto con una intencionalidad vacía, sino que siempre se está intencionando algo.

10. Según el diccionario enciclopédico Larousse (Tomo II, 1983, p. 428) "Parte superior de la bóveda craneal".

11. Hoy vemos el punto culmine de este proceso en el llamado "arte confesional", en el cual se exhibe la propia intimidad con diferentes matices. Por un lado, Tracey Emin utilizó como temas su violación, sus dos abortos y el tamaño del pene de su novio entre otras cosas. Por otro lado, el argentino Guillermo Iuso hizo de su obra un registro autobiográfico como si uno leyera viejos cuadernos de apuntes escritos con Silvapen.

\section{Bibliografía}

Adorno, T. (1983). Teoría estética, Madrid: Orbis.

Aulicino, J., "Un genio de luz y sombra” en Revista ñ, número 133 del sábado 14 de abril de 2006. Recuperado de: http://edant.clarin.com/suplementos/cultura/2006/04/15/u-01176154.htm Barker, F. S. (1984). Cuerpo y temblor. Un ensayo sobre la sujeción. Buenos Aires: Edit. Per Abbat.

Camus, A. (2004). El mito de Sísifo. Buenos Aires: Editorial Losada S. A.

Eagleton, T. (1998). Las ilusiones del posmodernismo. Buenos Aires: Paidos. 
Elías, N. (1994). El proceso de la civilización. México: Fondo de Cultura Económica. Foucault, M. (1987). Vigilar y Castigar, el nacimiento de la prisión. Buenos Aires: Siglo XXI. Grüner, E. El sitio de la mirada.

Jonas, H. (2000). El principio vida. Valladolid: Trotta.

Le Breton, D. (2002). Antropología del cuerpo y modernidad. Buenos Aires: Editorial Nueva Visión.

Parrondo, J. C. (2005). “Rembrandt. Sabiduría y emoción” en Los grandes genios del arte, Tomo número 11, Rembrandt, Biblioteca del diario El Mundo de España, Unidad Editorial S. A.

Rosenberg, J. (1987). Rembrandt. Vida y obra. Madrid: Alianza.

Unamuno, M. (1993). Del sentimiento trágico de la vida. España: Editorial Planeta - De Agostini.

\begin{abstract}
The production and context of the Dutch painter Rembrandt Harmenszoon van Rijn, allows an analysis of various changes in the conceptions of subjectivity and body that occur in the European world at the beginning of the 17th century. Through the so-called anatomy lessons and his self-portraits, Rembrandt gives an account of rationalization, the appearance of the individual and objectification of corporality; from a body united to the community in the Middle Ages, to the representation in the Modernity of a body as an inert object; from the community subject to the perception of the subject as a unit.
\end{abstract}

Keywords: subjectivity - Rembrandt - Harmenszoon van Rijn - 1606-1669 - art - Baroque mod.

Resumo: A produção e o contexto do pintor holandês Rembrandt Harmenszoon van Rijn, permite uma análise de várias mudanças nas concepções de subjetividade e do corpo que ocorrem no mundo europeu no início do século XVII. Através do chamadas de lições de anatomia e seus auto-retratos, Rembrandt dá conta da racionalização, a aparência do indivíduo e a objetivação da corporeidade: de um corpo unido à comunidade na a Idade Média, à representação na Modernidade de um corpo como um objeto inerte da comunidade sujeito à percepção do sujeito como uma unidade.

Palavras chave: Subjetividade - Rembrandt - Harmenszoon van Rijn - 1606-1669 - arte barroco - modernidade - estética.

[Las traducciones de los abstracts fueron supervisadas por el autor de cada artículo] 